\title{
On Throughput Optimality with Delayed Network-State Information
}

\author{
Lei Ying and Sanjay Shakkottai
}

\begin{abstract}
We study the problem of routing/scheduling in a wireless network with partial/delayed Network (channel and queue) State Information (NSI). We consider two cases: (i) centralized routing/scheduling, where a central controller obtains heterogeneous delayed information from each of the nodes (thus, the controller has NSI with different delays from different nodes), and makes the routing/scheduling decisions; (ii) decentralized routing/scheduling, where each node makes a decision based on its current channel and queue states along with homogeneous delayed NSI from other nodes.

For each of the cases (with additional flow restrictions for the decentralized routing/scheduling case), we first characterize the optimal network throughput regions under the above described NSI models and show that the throughput regions shrinks with the increase of delay. Further, we propose channel and queue length based routing/scheduling algorithms that achieve the above throughput regions.
\end{abstract}

\section{INTRODUCTION}

The wireless network provides a versatile platform to support a diverse set of applications such as voice, multimedia, data and messaging. These applications can exist over many configurations - citywide mesh networks, cellular deployments for mobile users, and ad-hoc battlefield networks. A key component in engineering such networks is the routing/scheduling algorithm. So far, most research focuses on developing routing/scheduling algorithms with complete Network State Information (NSI - the channel and queue state information of the entire network) by assuming that instantaneous NSI is available [1], [2], [3], [4], [5], [6], [7], [8], [9], [10], [11], or channel states are constant [12], [13], [14], [15], [16], [17]. We refer to [18], [19] for comprehensive surveys. While these algorithms optimize network performance with complete and instantaneous NSI; they could be inefficient in practical deployments because $(i)$ obtaining complete information could incur significant communication/computation overhead, and (ii) channel states could vary rapidly in a dynamic environment - thus, only delayed NSI might be available.

Notably, there have been recent studies on wireless scheduling with partial/delayed NSI. The decentralized channel-aware ALOHA has been developed in [20], [21], [22] for uplink networks, where each node transmits based on their own NSI. For downlink networks, the authors in [23] have considered

A shorter version of this paper appears in the Proceedings of the 2008 Information Theory and Applications Workshop [31]. L. Ying is with the Department of Electrical and Computer Engineering, Iowa State University. S. Shakkottai is with the Department of Electrical and Computer Engineering, The University of Texas at Austin, and with the Wireless Communications and Networking Group (WNCG). e-mail: leiyingeiastate.edu, shakkott dece.utexas. edu. a scenario where the base-station can only access the NSI of a subset of mobiles, and have developed a variant of the MaxWeight rule [2], which is throughput-optimal. Furthermore, the authors in [24], [25], [26], [27], [28] have taken account of the cost of channel probing, and have studied the trade-off between probing cost and scheduling gain. Joint channel-probing and transmission-scheduling algorithms have been developed to maximize network throughput. In [29], the authors have studied joint routing and scheduling with noisy channel estimates in the context of channels that vary i.i.d. across time. In [30], the authors consider a base-station connected to a collection of mobiles, and study the scheduling problem (uplink and downlink) when the channel and queue states are known periodically. They develop throughput optimal policies where a (matching) decision is made at each slot based on this globally known delayed information.

While partial/delayed NSI has been studied in the prior work, there are two distinguishing features in our study. First, we study the case of a general network topology with heterogeneous delays from each of the nodes in the network to the central controller (the decision maker). Second, and perhaps more important, we study the case of decentralized decision making where each node makes a transmission decision based on locally known information, and the information known at each node is different (i.e., different nodes have different "views" of the network). The resulting information inconsistency can cause nodes to make potentially conflicting decisions (e.g., two nodes on the same collision domain may decide to transmit simultaneously because they have different delayed versions of the channel-states and cannot coordinate their decisions with each other instantaneously). The two cases to be considered in this paper are summarized below:

(i) There is a central controller that selects a subset of links to transmit based on heterogeneous delayed NSI at each time slot.

(ii) There is no central controller, and each node has to make transmission decision based on their own instantaneous NSI and delayed NSI from other nodes. Uplink networks and ad-hoc networks are such examples. In this scenario, on one hand, the nodes have inconsistent views of the network because they have different local NSI; but on the other hand, we need to exploit the local instantaneous NSI since it provides more accurate network information. Developing a consistent policy based on the inconsistent information is the key challenge in this scenario.

In both of these contexts, we study routing/scheduling 
algorithms with delayed NSI. The main contributions of this paper include:

(i) We characterize the network throughput regions with delayed NSI for both the centralized and decentralized cases (under additional assumptions on delays and flows, see Section V). We present examples in Section IV and Section V where we explicitly compute the throughputoptimal policy. We will see from these examples that the network throughput region is determined by the available NSI, and shrinks with the increase of the delay in NSI.

(ii) We also develop throughput-optimal routing/scheduling algorithms based on the delayed NSI. For the centralized case, the algorithm is a variant of the back-pressure algorithm proposed in [1], which uses the expected channelstates, conditioned on the delayed channel-states, in the routing/scheduling. For the decentralized case, the proposed algorithm contains two parts: each node first calculates a threshold vector based on the global delayed NSI; and then makes a transmission decision based on the local instantaneous NSI and the threshold vector.

We finally remark that optimal control with information delays has been studied since the classic work of Witsenhausen [32], [33], [34], [35]; in general it is known to be hard to characterize the optimal policy in a delayed/decentralized setting. However, in this paper, we are interested in determining optimal stabilizing policies, which is a weaker requirement than minimizing a cost function; and we are able to characterize such stabilizing policies in several settings.

\section{BASIC MODEL}

We first introduce the basic models in this section.

Network model: Consider a network represented by a graph $\mathcal{G}=(\mathcal{N}, \mathcal{L})$, where $\mathcal{N}$ is the set of nodes and $\mathcal{L}$ is the set of directed links (channels). Denote by $(m, n)$ a link from node $m$ to node $n$, where $m$ is the transmitter and $n$ is the receiver.

Traffic model: We let $[s, d]$ denote a flow in the network, where $s$ denotes the source of the flow, and $d$ denotes the destination of the flow. Further, let $\mathcal{F}$ denote the set of all flows in the network. Assume that time is discretized. Denote by $A_{[s, d]}[t]$ the number of packets injected into node $s$ and destined to node $d$ at time slot $t$. We assume that $\left\{A_{[s, d]}[t]\right\}_{[s, d] \in \mathcal{F}}$ are stationary random variables independent across time and flows, $\mathbf{E}\left[A_{[s, d]}[t]\right]=a_{[s, d]}$, and $A_{[s, d]}[t] \leq A_{\max }$ for all $[s, d] \in \mathcal{F}$ and $t$. A network with four nodes, four links, and three flows is illustrated in Figure 1.

Given traffic $\left\{A_{[s, d]}[t]\right\}_{[s, d] \in \mathcal{F}}$ and a routing/schedulig policy, we say the network is stochastically stable if the mean of the sum of the queue-lengths is bounded. Traffic $\left\{A_{[s, d]}[t]\right\}_{[s, d] \in \mathcal{F}}$ is said to be supportable if there exists any routing/scheduling policy (without packet dropping), under which the network is stochastically stable.

Channel-fading model: We denote by $C_{(m, n)}[t]$ the state of channel $(m, n)$ at time slot $t$, i.e., the number of packets that can be transmitted over link $(m, n)$ during time slot $t$. We assume that $C_{(m, n)}[t]$ is random and can be represented by a

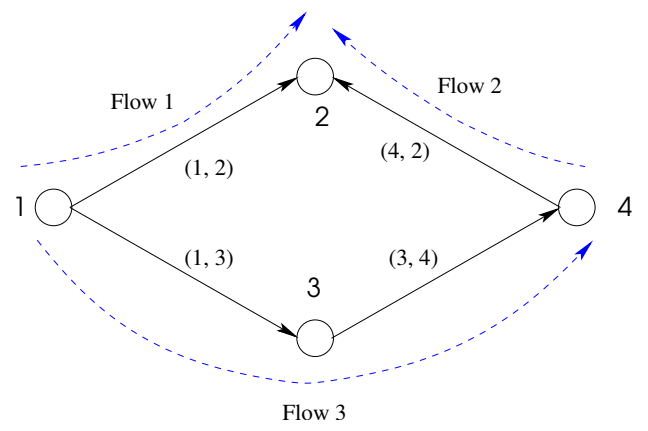

Fig. 1. A network example

finite-state Markov chain ${ }^{1}$, i.e.,

$$
\begin{aligned}
& \operatorname{Pr}\left(C_{(m, n)}[t] \mid C_{(m, n)}[t-1], \ldots, C_{(m, n)}[0]\right) \\
= & \operatorname{Pr}\left(C_{(m, n)}[t] \mid C_{(m, n)}[t-1]\right) .
\end{aligned}
$$

and $C_{(m, n)}[t] \in \mathcal{C}$ for $|\mathcal{C}|<\infty$. We assume that $C_{(m, n)}[t] \leq$ $C_{\max }$ for all $(m, n) \in \mathcal{L}$ and $t$. The one-step transition probabilities of the Markov chains are assumed to be known at all nodes, and $\left\{C_{(m, n)}[t]\right\}_{(m, n) \in \mathcal{L}}$ are independent across links.

Interference model: We assume a general collision model in this paper. If two links interfere with each other, simultaneous transmissions on the two links will lead to a collision and no information (packet) can get through. For link $(m, n)$, we denote by $\mathcal{I}_{(m, n)}$ the set of links that interfere with link $(m, n)$. For example, in downlink/uplink networks, where only one link can successfully transmit at a time, $\mathcal{I}_{(m, n)}=\mathcal{L} \backslash\{(m, n)\}$.

Queue dynamics: We assume that each node maintains a separate queue for each destination. Denote by $Q_{<m, d>}[t]$ the length of the queue maintained at node $m$ for destination $d$. It has been shown in [2] that per-destination queues are sufficient for stability. In most of this paper, we assume that hop-by-hop feedback is available so that node $m$ knows those packets successfully transmitted over link $(m, n)$, and immediately removes them from the queues. Thus, the dynamics of queue $<m, d>$ can be described as follows:

$$
\begin{aligned}
Q_{<m, d>}^{\mathcal{P}}[t+1]= & Q_{<m, d>}^{\mathcal{P}}[t]+A_{[m, d]}[t]+ \\
& \sum_{(k, m) \in \mathcal{L}} \nu_{(k, m), d}^{\mathcal{P}}[t]-\sum_{(m, n) \in \mathcal{L}} \nu_{(m, n), d}^{\mathcal{P}}[t],
\end{aligned}
$$

where $\nu_{(m, n), d}^{\mathcal{P}}[t]$ is the number of packets transmitted over link $(m, n)$ at time $t$ with destination $d$. The superscript $\mathcal{P}$ indicates the policy used. (However, for one of the cases to be considered, somewhat different queue dynamics will be assumed, which will be described explicitly when the case is presented).

\section{MORE NOTATIONS}

To simplify our notations, we let $\mathbf{C}[t]$ denote the states of all channels at time slot $t$, i.e.,

$$
\mathbf{C}[t] \triangleq\left\{C_{(m, n)}[t]\right\}_{(m, n) \in \mathcal{L}} .
$$

\footnotetext{
${ }^{1}$ In this paper, whenever there is no ambiguity, we abuse notation to let $\operatorname{Pr}\left(C_{(m, n)}[t] \mid C_{(m, n)}[t-1]\right):=\operatorname{Pr}\left(C_{(m, n)}[t]=c_{1} \mid C_{(m, n)}[t-1]=c_{2}\right)$ (i.e., we do not explicitly indicate the values the random variables take in conditional probability expressions).
} 
We also let $\mathbf{C}[t]\left(\tau_{1}: \tau_{2}\right)$ denote the states of all channels from time slot $t-\tau_{2}$ to $t-\tau_{1}\left(\tau_{2} \geq \tau_{1}\right)$, i.e.,

$$
\mathbf{C}[t]\left(\tau_{1}, \tau_{2}\right) \triangleq\left\{C_{(m, n)}[t-s]\right\}_{(m, n) \in \mathcal{L}, s \in\left[\tau_{1}, \tau_{2}\right]} .
$$

Similarly, we define $\mathbf{Q}[t]$ and $\mathbf{Q}[t]\left(\tau_{1}, \tau_{2}\right)$.

If the network is stochastically stable under routig/scheduling policy $\mathcal{P}$, we denote by $\mathbf{C}[\infty]$ and $\mathbf{Q}^{\mathcal{P}}[\infty]$ the steady-state channel and queue states under policy $\mathcal{P}$. We further define

$$
\mathbf{C}\left(\tau_{1}, \tau_{2}\right) \triangleq \lim _{t \rightarrow \infty}\left\{C_{(m, n)}[t-s]\right\}_{(m, n) \in \mathcal{L}, s \in\left[\tau_{1}, \tau_{2}\right]},
$$

and $\mathbf{Q}^{\mathcal{P}}\left(\tau_{1}, \tau_{2}\right)$ is defined similarly.

Furthermore, let $D_{(m, n)}^{\mathcal{P}}[t]$ denote the transmission decision on link $(m, n)$ under policy $\mathcal{P}$, i.e., $D_{(m, n)}^{\mathcal{P}}[t]=1$ if node $m$ attempts to transmit over link $(m, n)$ at time slot $t$; and $D_{(m, n)}^{\mathcal{P}}[t]=0$ otherwise. We also let $S_{(m, n)}^{\mathcal{P}}[t]$ denote the achievable rate over link $(m, n)$ at time $t$. Assuming that a node can only transmit over one link at a time, we have

$$
S_{(m, n)}^{\mathcal{P}}[t]=C_{(m, n)}[t] D_{(m, n)}^{\mathcal{P}}[t] \prod_{(k, l) \in \mathcal{I}_{(m, n)}}\left(1-D_{(k, l)}^{\mathcal{P}}[t]\right) .
$$

Note that $S_{(m, n)}^{\mathcal{P}}[t] \geq \nu_{(m, n)}^{\mathcal{P}}[t]$, where the strict inequality holds when there is no enough packets in the queue.

Finally, we say a set of links $\mathcal{M}$ is an independent-set if for any link $(m, n) \in \mathcal{M}, \mathcal{I}_{(m, n)} \cap \mathcal{M}=\emptyset$.

\section{Centralized Routing/Scheduling With Delayed NSI: An Example and The Main Result}

We first consider the centralized case where the central controller knows $\mathbf{C}[t-\boldsymbol{\tau}]$ and $\mathbf{Q}[t-\boldsymbol{\tau}]$, and makes routing/scheduling decisions based on this delayed $\mathrm{NSI}^{2}$. Here, we abuse notation and let $\tau$ denote the vector of delays. In particular, $\boldsymbol{\tau}=\left\{\tau_{(m, n)}\right\}_{(m, n) \in \mathcal{L}}$ when associated with the channel-states and $\boldsymbol{\tau}=\left\{\tau_{<m, d>}\right\}_{m, d \in \mathcal{N}}$ when associated with the queue-states. We also assume that the routing/scheduling decisions are executed in the network immediately without delay.

\section{A. An Illustrative Example}

Consider a wireless downlink network with two nodes and a single base-station as shown in Figure 2 . Denote by $(0,1)$ the channel from the base-station to node 1 and by $(0,2)$ the channel from the base-station to node 2. Assume that the channels are represented by the two-state Markov chain as shown in Figure 3.

We consider two different NSI structures:

\footnotetext{
${ }^{2}$ In this paper, we assume that the central controller knows the channel and queue trajectories with some (node dependent) delay. This model can easily be relaxed to the case where as before, the controller knows the channels with delay; however, it knows the queue lengths only roughly periodically with (node-dependent) delay. In other words, at each time $t$ and for each queue $Q_{<m, d>}$, it suffices for the controller to know the queue length only at some time $s$ in the past (as opposed to the entire trajectory) in the finite interval $s \in\left(t-\bar{d}_{<m, d>}, t-\underline{\mathrm{d}}_{<m, d>}\right)$, where $0 \leq \tau_{m, n} \leq \underline{\mathrm{d}}_{<m, d>} \leq$ $\bar{d}_{<m, d>}<\infty$. However, for notational ease, in this paper, we only discuss the case where $\underline{\mathrm{d}}_{<m, d>}=\bar{d}_{<m, d>}=\tau_{<m, d>}$.
}

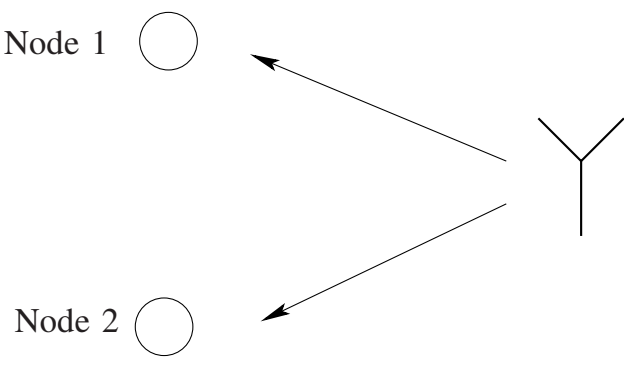

Fig. 2. A downlink network

0.2

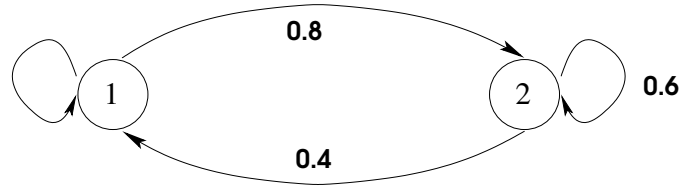

State 1: 50 packets/time slot

State 2: 0 packets/time slot

Fig. 3. A two-state Markov chain

(i) The base-station has the instantaneous NSI. In this case, the network throughput region is the region depicted using the "+"-line in Figure 4. The following queuelength-based throughput-optimal scheduling algorithm has been proposed in [1]: At time slot $t$, the base-station transmits to node $i^{*}[t]$ such that

$$
i^{*}[t]=\arg \max _{i=1,2} Q_{<0, i>}[t] C_{(0, i)}[t] .
$$

(ii) The base-station has one time slot delayed NSI, i.e., the base-station only has $C_{(0, i)}[t-1]$ and $Q_{<0, i>}[t-1]$. Then the network throughput region is the region depicted using the " $\times$ "-line Figure 4. A throughput optimal policy is as follows: At time slot $t$, the base-station transmits to node $i^{*}[t]$ such that

$$
i^{*}[t]=\arg \max _{i=1,2} Q_{<0, i>}[t-1] \mathbf{E}\left[C_{(0, i)}[t] \mid C_{(0, i)}[t-1]\right] .
$$

The throughput optimality of the algorithm above will be proved in Section VI.

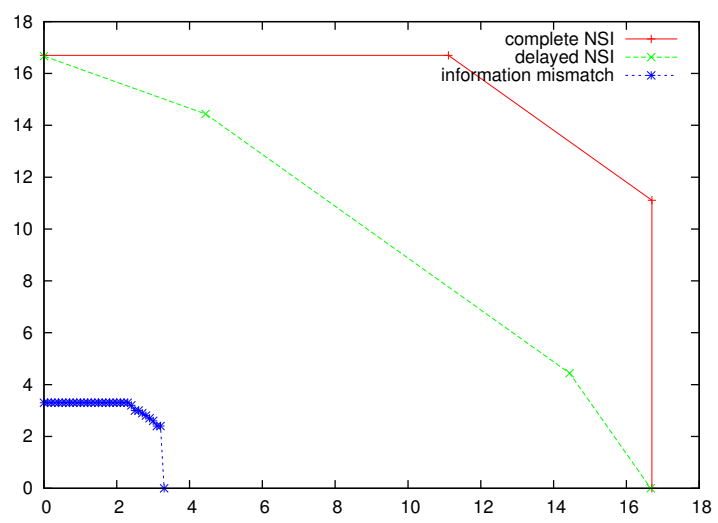

Fig. 4. The throughput regions of the downlink network 
Remark 1: Comparing case (i) and (ii), we can see that the delay in NSI leads to a throughput degradation. The intuition is easy to understand. While, without delay, the base-station can transmit 50 packets/slot over link $(0, i)$ when $C_{(0, i)}[t]=50$; only 10 packets/slot in average can be successfully transmitted if the base-station does not have $C_{(0, i)}[t]$, but only knows $C_{(0, i)}[t-1]=50$. With the one-time-slot delay, the channels revealed to the base-station can be modeled as the Markov chain illustrated in Figure 5. Such a channel twist due to information delay reduces the achievable throughput region. We remark that qualitatively similar observations have been made in the context of multi-channel access point networks for uplink and downlink scheduling ${ }^{3}$ in [30].

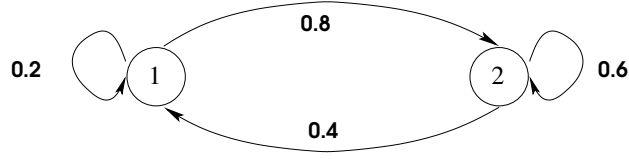

State 1: 10 packets/time slot $\quad$ State 2: 20 packets/time slot

Fig. 5. The twisted two-state Markov chain

Remark 2: Furthermore, we would like to comment that incorporating the delay in scheduling is critical to achieve the maximum network throughput. For example, we could simply treat the delayed NSI as the instantaneous NSI, and use the following naive algorithm: At time slot $t$, the base-station transmits to node $i^{*}[t]$ such that

$$
i^{*}[t]=\arg \max _{i=1,2} Q_{<0, i>}[t-1] C_{(0, i)}[t-1] .
$$

Then, the achievable throughput region is the region depicted using the "*"-line in Figure 4 (this throughput region is obtained using a simulation with constant arrivals). We can see that this information mismatch (using the delayed NSI as the current NSI) leads to a significant performance loss.

\section{B. Main Result}

For the centralized scenario, we propose the following onoff routing/scheduling based on conditional expectation.

On-Off Routing/Scheduling: At time slot $t$,

(1) The controller first computes the optimal independentlink-set $\mathcal{M}^{*}[t]$ which maximizes

$$
\sum_{(m, n) \in \mathcal{M}} \mathbf{E}\left[C_{(m, n)}[t] \mid C_{(m, n)}\left[t-\tau_{(m, n)}\right]\right] P_{(m, n)}[t],
$$

where

$$
\begin{aligned}
P_{(m, n)}[t]=\max _{d \in \mathcal{N}} & \left(Q_{<m, d>}\left[t-\tau_{<m, d>}\right]\right. \\
& \left.-Q_{<n, d>}\left[t-\tau_{<n, d>}\right]\right),
\end{aligned}
$$

\footnotetext{
${ }^{3}$ The model studied in [30] is in the context where the base-station (a central controller) periodically gets all channel and queue states together (homogeneous delay).
}

(2) Node $m$ transmits the packets from queue $d_{(m, n)}^{*}[t]$ over link $(m, n)$ with a rate $C_{(m, n)}[t]$ if $(m, n) \in \mathcal{M}^{*}[t]$, where

$$
\begin{aligned}
d_{(m, n)}^{*}[t]=\arg \max _{d} & \left(Q_{<m, d>}\left[t-\tau_{<m, d>}\right]\right. \\
& \left.-Q_{<n, d>}\left[t-\tau_{<n, d>}\right]\right) .
\end{aligned}
$$

We will prove in Section VI that this on-off routing/scheduling is throughput-optimal if the delays in channel feedbacks are smaller than the delays in queue feedbacks.

Theorem 1: Given the delays satisfying $\max _{(m, n) \in \mathcal{L}} \tau_{(m, n)}<$ $\min _{m, d \in \mathcal{N}} \tau_{<m, d>}$ and traffic $\mathbf{A}[t]$ such that $(1+\epsilon) \mathbf{A}[t]$ is supportable, the network is stochastically stable under the on-off routing/scheduling algorithm.

\section{Decentralized Routing/Scheduling With DELAYED NSI: EXAMPLES AND MAIN RESUltS}

In this section, we consider the decentralized scenario where a node has its own local instantaneous NSI and a networkwide delayed NSI. Thus, the nodes have different views of the network. The challenge is to develop a consistent policy based on the inconsistent NSI.

\section{A. Illustrative Example: A Single-hop Network}

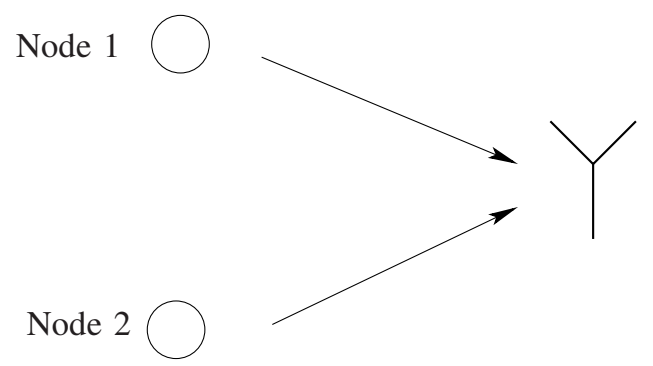

Fig. 6. Uplink network

Consider an uplink network with two nodes and a single base-station as illustrated in Figure 6. The channels between the base-station and the nodes are each described by a twostate Markov chain as illustrated in Figure 3.

In this example, we assume that each of the two nodes has exact knowledge of its channel and queue state (over the current and previous time-slots), but has one-time-slot delay in the information from the other node. Equivalently, both of the nodes have delayed NSI $\left\{C_{(i, 0)}[t-1], Q_{<i, 0>}[t-1]\right\}_{i=1,2}$, and additionally, each node $i$ only knows $C_{(i, 0)}[t]$ and $Q_{<i, 0>}[t]$.

In this case, the throughput region is the region depicted using the " $\times$ "-line in Figure 7. A throughput optimal policy is as follows: At time slot $t$,

(1) Node 1 and 2 first calculate a threshold vector $\mathbf{T}^{*}[t]$ by solving the optimization problem (3).

(2) Node $i$ transmits to the base-station if $C_{(i, 0)}[t] \geq$ $T_{(i, 0)}^{*}[t]$.

Remark 1: The algorithm above consists of two steps: 


$$
\begin{array}{r}
\mathbf{T}^{*}[t]=\arg \max _{\mathbf{T}} Q_{<1,0>}[t-1] \mathbf{E}\left[C_{(1,0)}[t] \mathbf{1}_{C_{(1,0)}[t] \geq T_{(1,0)}, C_{(2,0)}[t]<T_{(2,0)}} \mid \mathbf{C}[t-1]\right]+ \\
Q_{<2,0>}[t-1] \mathbf{E}\left[C_{(2,0)}[t] \mathbf{1}_{C_{(2,0)}[t] \geq T_{(2,0)}, C_{(1,0)}[t]<T_{(1,0)}} \mid \mathbf{C}[t-1]\right]
\end{array}
$$

(1) Determine the threshold vector based on global delayed NSI.

(2) Make the transmission decisions based on local instantaneous (current) NSI.

Remark 2: This algorithm is a combination of greedycontention scheduling and collision-avoidance scheduling. For example, given $C_{(1,0)}[t-1]=C_{(2,0)}[t-1]=50$,

- If $Q_{<1,0>}[t-1]>4 Q_{<2,0>}[t-1]$, then $\mathbf{T}^{*}[t]=[0,51]$. Thus, node 2 keeps silent, and node 1 attempts to transmit. This corresponds to a "conservative" collisionavoidance scheduling, where node 2 does not transmit irrespective of its current local channel state (i.e, $C_{(2,0)}[t]$ ). On the other hand, node 1 transmits (successfully) only if $C_{(1,0)}[t]=50$.

- If $Q_{<1,0>}[t-1] / Q_{<2,0>}[t-1] \in[0.25,4]$, then $\mathbf{T}^{*}[t]=$ $[50,50]$. In this case, both nodes attempt to transmit when $C_{(i, 0)}[t]=50, i=1,2$. Further, if any one of the users' current channel is in the 0 state, that node does not attempt to transmit. This corresponds to a greedy contention scheduling mechanism, where the randomization is provided by the channel (i.e., both channels are in state 50 in the previous time-slot, and there is a positive probability for each of the four cases $(50,50),(50,0)(0,50,(0,0)$ to occur in the current timeslot).

Remark 3: We also consider a strategy where we ignore the delays in NSI, and use the algorithm that is optimal with the complete and instantaneous NSI: Node 1 attempts to transmit if $C_{(1,0)}[t] Q_{(1,0)}[t]>C_{(2,0)}[t-1] Q_{(2,0)}[t-1]$, and node 2 attempts to transmit if $C_{(2,0)}[t] Q_{(2,0)}[t] \geq C_{(1,0)}[t-$ $1] Q_{(1,0)}[t-1]$. Assuming constant arrivals, the throughput region is obtained using the simulation, and depicted using " $\square$ "line in Figure 7. We can see that the information mismatch leads to a significant performance loss.

To illustrate the impact of delayed NSI on the network throughput, we also show the throughput region of the following two scenarios in Figure 7.

(i) Both nodes have $\left\{C_{(i, 0)}[t], Q_{<i, 0>}[t]\right\}_{i=1,2}$. The throughput region is depicted using the "+"-line.

(ii) Both nodes have $\left\{C_{(i, 0)}[t-1], Q_{<i, 0>}[t-1]\right\}$, but no instantaneous NSI is available. The throughput region is depicted using the " $*$ "-line.

We can see that the network throughput region shrinks with the increase of the delay. Thus, to maximize the network performance under delayed NSI, it is important that we understand the impact of the delayed NSI, and intelligently use the delayed NSI in routing/scheduling.

\section{B. Illustrative Example: A Multi-hop Network}

To further demonstrate the impact of delayed NSI. We consider a two-hop network with two flows as in Figure 8.

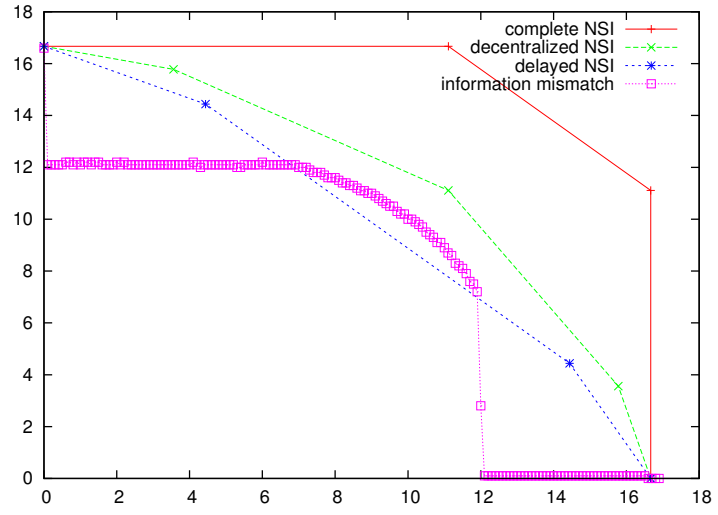

Fig. 7. The throughput regions of the single-hop network

We consider two NSI structures:

(i) The complete NSI is available at node 1 and 2 . The network throughput region is depicted using the "+"-line in Figure 9.

(ii) Node 1 has $C_{(1,2)}[t], Q_{<1,2>}[t], Q_{<1,3>}[t], C_{(2,3)}[t-1]$, and $Q_{<2,3>}[t-1]$. Node 2 has $C_{(2,3)}[t], Q_{<2,3>}[t]$, $C_{(1,2)}[t-1], Q_{<1,2>}[t-1]$, and $Q_{<1,3>}[t-1]$. The network throughput region is depicted using the " $\times$ "-line in Figure 9. In this scenario, the throughput-optimal algorithm can be viewed as a "back-pressure" like algorithm [1], [2] which determines the policy based on consistent delayed NSI and makes transmission decisions based on the best available NSI (we refer to Section V-C and (4) for additional details).

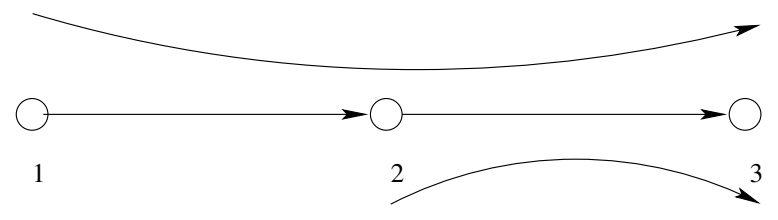

Fig. 8. Two-hop network

Remark: We also consider an information mismatch scenario, where we use the algorithm that is optimal with NSI structure (i) for NSI structure (ii). Assuming constant arrivals, the throughput region is obtained using the simulation, and depicted using " *"-line in Figure 7. We can see that such a information mismatch also leads to a performance loss.

\section{Main Result}

We now study multi-hop networks, where each node has its local instantaneous NSI, and homogeneous delayed NSI from other nodes. We propose the following threshold-based routing/scheduling algorithm. 


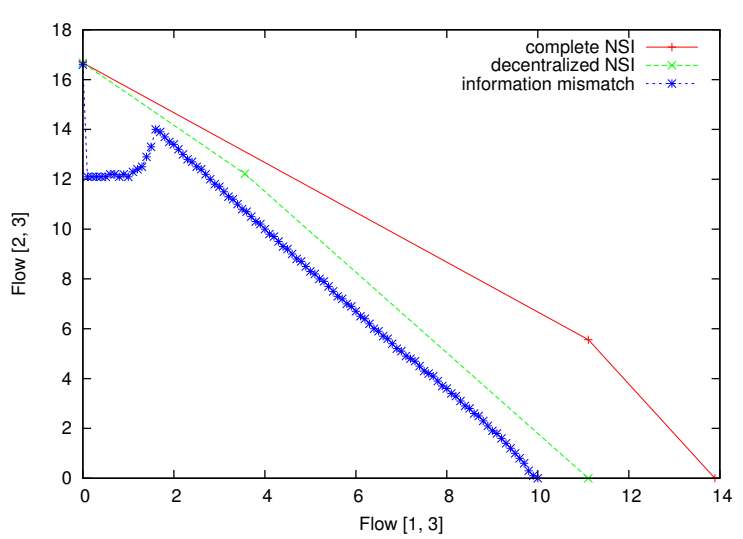

Fig. 9. Two-hop network: Throughput regions

Threshold-Based Routing/Scheduling: At time slot $t$,

(1) All nodes first compute a common threshold vector $\mathbf{T}^{*}[t]$ by solving the optimization problem (4).

(2) Node $m$ attempts to transmit packets from queue $d_{m}^{*}[t]$ over link $(m, n)$ if $C_{(m, n)} \geq T_{(m, n)}^{*}[t]$, where

$d_{(m, n)}^{*}[t]=\arg \max _{d}\left(Q_{<m, d>}[t-\tau]-Q_{<n, d>}[t-\tau]\right)$.

We consider two different NSI structures:

$S 1$ Node $m$ has $\left(C_{(m, n)}[t], Q_{<m, d>}[t]\right)$ for all $(m, n) \in \mathcal{L}$ and $d \in \mathcal{N}$, and $\left(C_{(l, k)}[t-1], Q_{<l, d>}[t-1]\right)$ for all $(l, k) \in \mathcal{L}$ for all $l, d \in \mathcal{N}$. In other words, each node has their instantaneous local NSI, and one time slot delayed NSI of the entire network.

$S 2$ All nodes have $\mathbf{C}[t-\tau]$ and $\mathbf{Q}[t-\tau]$. Node $m$ also has $C_{(m, n)}[t](0: \tau-1)$ and $Q_{<m, d>}[t](0: \tau-1)$ for $(m, n) \in \mathcal{L}$ and $d \in \mathcal{N}$. We assume that all flows are single-hop flows, and a transmitter is disjoint from other transmitters and receivers. It is easy to see that only a single queue is needed for each flow. Furthermore, we assume that transmitters cannot detect collisions (it needs to explicitly wait for an acknowledgment (ACK) from the receiver to determine success/failure of the transmission), and a source does not delete transmitted packets until it receives an acknowledgment from the destination (after which it can flush packets in its queue corresponding to the successful ACKs). Thus, the queue dynamics can be described as follows:

$$
\begin{gathered}
Q_{<m, d>}[t+1] \\
=\left(Q_{<m, d>}[t]+A_{[m, d]}[t]-\nu_{<m, d>}\left[t-\tau_{f}\right]\right)^{+},
\end{gathered}
$$

where $\tau_{f}$ is the feedback delay, and assumed to be larger than $\tau$.

The main result, given the two NSI structures above, is as follows.

Theorem 2: Given NSI structure $S 1$ or $S 2$, and traffic $\mathbf{A}[t]$ such that $(1+\epsilon) \mathbf{A}[t]$ is supportable, the network is stochastically stable under the threshold-based routing/scheduling algorithm.

\section{Vi. Centralized Algorithm with Delayed NSI: DETAILED ANALYSIS}

\section{A. Throughput Region}

In this subsection, we will characterize the optimal throughput region with a central controller and delayed NSI. First, given

$$
\mathbf{C}[0]=\left\{C_{(m, n)}[0]\right\}_{(m, n) \in \mathcal{L}}
$$

and an independent-link-set $\mathcal{M}$, we define

$$
\begin{aligned}
& \mathbf{S}(\mathbf{C}[0], \mathcal{M}) \\
= & \left\{S_{(m, n)}\left(C_{(m, n)}[0], \mathcal{M}\right)\right\}_{(m, n) \in \mathcal{L}} \\
= & \left\{\mathbf{E}\left[C_{(m, n)}\left[\tau_{(m, n)}\right] \mid C_{(m, n)}[0]\right] \mathbf{1}_{(m, n) \in \mathcal{M}}\right\}_{(m, n) \in \mathcal{L}} .
\end{aligned}
$$

Further, we define $\eta(\mathbf{C}[0])$ to be the convex hull of $\mathbf{S}(\mathbf{C}[0], \mathcal{M})$ over all independent-link set $\mathcal{M}$ :

$$
\eta(\mathbf{C}[0])=\mathcal{C H}_{\mathcal{M}}(\mathbf{S}(\mathbf{C}[0], \mathcal{M})) .
$$

Then, we define

$\Lambda_{\tau}=\left\{\boldsymbol{\eta}: \boldsymbol{\eta}=\sum_{\mathbf{c} \in \mathcal{C}|\mathcal{L}|} \operatorname{Pr}(\mathbf{C}[0]=\mathbf{c}) \boldsymbol{\eta}_{\mathbf{c}}, \boldsymbol{\eta}_{\mathbf{c}} \in \eta(\mathbf{C}[0]=\mathbf{c})\right\}$.

In the following analysis, we will prove that $\Lambda_{\tau}$ is the optimal throughput region. To simplify our notation, we define

$$
\mathbf{C}[t-\boldsymbol{\tau}]=\left\{C_{(m, n)}\left[t-\tau_{(m, n)}\right]\right\}_{(m, n) \in \mathcal{L}}
$$

and

$$
\mathbf{Q}[t-\boldsymbol{\tau}]=\left\{Q_{<m, d>}\left[t-\tau_{<m, d>}\right]\right\}_{m, d \in \mathcal{N}} .
$$

Furthermore, we define

$$
\mathbf{Y}[t]=\left\{Q_{<m, d>}[t]\left(0: \tau_{<m, d>}\right), C_{(m, n)}[t]\left(0: \tau_{(m, n)}\right)\right\}
$$

for all $m, d \in \mathcal{N}$ and $(m, n) \in \mathcal{L}$. It is easy to see that $\mathbf{Y}[t]$ is Markovian. Now, given arrivals $\mathbf{A}[t]=\left\{A_{[s, d]}[t]\right\}_{[s, d] \in \mathcal{F}}$, the network is stochastically stable under a scheduling policy $\mathcal{P}$ if $\mathbf{Y}^{\mathcal{P}}[t]$ is positive recurrent, where the superscript indicates the scheduling policy. Thus, given a stabilizing policy $\mathcal{P}$, we define the steady-state distribution $\mathbf{Y}^{\mathcal{P}}[\infty]$ as follows:

$$
\begin{aligned}
& \left\{\left(Q_{<m, d>}^{\mathcal{P}}\left(0: \tau_{<m, d>}\right), C_{(m, n)}\left(0: \tau_{(m, n)}\right)\right)\right\} \\
\triangleq & \lim _{t \rightarrow \infty}\left\{Q_{<m, d>}^{\mathcal{P}}[t]\left(0: \tau_{<m, d>}\right), C_{(m, n)}[t]\left(0: \tau_{(m, n)}\right)\right\} \\
= & \lim _{t \rightarrow \infty} \mathbf{Y}^{\mathcal{P}}[t] \\
= & \mathbf{Y}^{\mathcal{P}}[\infty] .
\end{aligned}
$$

Now denote by $\mathbf{D}^{\mathcal{P}}(\mathbf{Q}[t-\tau]=\mathbf{q}, \mathbf{C}[t-\boldsymbol{\tau}]=\mathbf{c})$ the decision under policy $\mathcal{P}$ given delayed NSI $(\mathbf{Q}[t-\boldsymbol{\tau}]=$ $\mathbf{q}, \mathbf{C}[t-\tau]=\mathbf{c})$. Based on a stabilizing policy $\mathcal{P}$, we now define a corresponding (probabilistic) time-sharing policy $\mathcal{P}_{s}$ which uses the knowledge of the steady-state distribution of the queue lengths and channel states under policy $\mathcal{P}$ (denoted as before by $\mathbf{Y}^{\mathcal{P}}[\infty]$ ) along with the delayed channel states $\mathbf{c}$ as follows:

Time-sharing Policy $\mathcal{P}_{s}$ : Given the delayed channel-state information $\mathbf{C}[t-\boldsymbol{\tau}]=\mathbf{c}$, we let

$$
r_{\mathbf{q} \mid \mathbf{c}}^{\mathcal{P}}=\operatorname{Pr}(\mathbf{Q}(\boldsymbol{\tau}: \boldsymbol{\tau})=\mathbf{q} \mid \mathbf{C}(\boldsymbol{\tau}: \boldsymbol{\tau})=\mathbf{c}) .
$$




$$
\begin{aligned}
\mathbf{T}^{*}[t]=\arg \max _{\mathbf{T}} & \sum_{(m, n) \in \mathcal{L}} \max _{d}\left(Q_{<m, d>}[t-\tau]-Q_{<n, d>}[t-\tau]\right) \times \\
& \mathbf{E}\left[C_{(m, n)}[t] \mathbf{1}_{C_{(m, n)}[t] \geq T_{(m, n)}} \Pi_{(l, k) \in \mathcal{I}_{(m, n)}} \mathbf{1}_{C_{(l, k)}[t]<T_{(l, k)}} \mid \mathbf{C}[t-\tau]\right] .
\end{aligned}
$$

Then, at each time when the delayed channel state is $\mathbf{C}[t-\boldsymbol{\tau}]=\mathbf{c}$, the policy $\mathcal{P}^{s}$ probabilistically makes decisions (denoted by $\mathbf{D}^{\mathcal{P}_{s}}$ ) as follows:

Let $\mathcal{Q}=\left\{\mathbf{q}: r_{\mathbf{q} \mid \mathbf{c}}^{\mathcal{P}}>0\right\}$. The decision is to choose

$\mathbf{D}^{\mathcal{P}_{s}}(\mathbf{C}[t-\tau]=\mathbf{c})=\mathbf{D}^{\mathcal{P}}(\mathbf{Q}[t-\tau]=\mathbf{q}, \mathbf{C}[t-\tau]=\mathbf{c})$

with probability $r_{\mathbf{q} \mid \mathbf{c}}^{\mathcal{P}}$.

In other words, for each delayed channel state $\mathbf{c}$, the policy $\mathcal{P}_{s}$ first randomly (with probability $r_{\mathbf{q} \mid \mathbf{c}}^{\mathcal{P}}$ ) picks a possible steady-state queue lengths under policy $\mathcal{P}$ and uses the resulting pair (q, c) as the "input" to policy $\mathcal{P}$ (which is known to be stabilizing) in order to get a decision. We note that this rule is in the same spirit as the Static Service Split (SSS) Rule in [3].

Next we will show that the service rates obtained under $\mathcal{P}$ or $\mathcal{P}_{s}$ are the same.

Lemma 3: Assume the delays satisfying $\max _{(m, n) \in \mathcal{L}} \tau_{(m, n)}<$ $\min _{k, d \in \mathcal{N}} \tau_{<k, d>}$. Conditioned on $\mathbf{C}[t-\boldsymbol{\tau}], Q_{<k, d>}\left[t-\tau_{<k, d>}\right]$ and $C_{(m, n)}[t-s]$ are independent for any $s<\tau_{(m, n)}$, $(m, n) \in \mathcal{L}$, and $k, d \in \mathcal{N}$.

Proof: Note that $Q_{<k, d>}\left[t-\tau_{<k, d>}\right]$ is determined by $\mathbf{C}[t]\left(\tau_{<k, d>}, t\right), \mathbf{A}[t]\left(\tau_{<k, d>}, t\right)$, and $\mathbf{D}^{\mathcal{P}}[t]\left(\tau_{<k, d>}, t\right)$, where

$$
\mathbf{D}^{\mathcal{P}}[t]=\mathbf{D}^{\mathcal{P}}(\mathbf{Q}[t-\tau], \mathbf{C}[t-\boldsymbol{\tau}])
$$

It can be easily verified that the lemma holds when $\max _{(m, n) \in \mathcal{L}} \tau_{(m, n)}<\min _{k, d \in \mathcal{N}} \tau_{<k, d>}$

Lemma 4: Given $\max _{(m, n) \in \mathcal{L}} \tau_{(m, n)}<\min _{k, d \in \mathcal{N}} \tau_{<k, d>}$ and policy $\mathcal{P}$ that stabilizes traffic $\mathbf{A}[t]$, we have that

$$
\lim _{t \rightarrow \infty} \mathbf{E}\left[S_{(m, n)}^{\mathcal{P}}[t]\right]=\lim _{t \rightarrow \infty} \mathbf{E}\left[S_{(m, n)}^{\mathcal{P}_{s}}[t]\right],
$$

where $\mathcal{P}_{s}$ is the corresponding time-sharing policy.

Proof: The lemma is proved in(6), where equality $(a)$ holds due to Lemma 3, and equality $(b)$ yields from the definition of the time-sharing policy $\mathcal{P}_{s}$.

Lemma 5: Consider the centralized case where the central controller makes routing/scheduling decisions based on $\mathbf{C}[t-$ $\boldsymbol{\tau}]$ and $\mathbf{Q}[t-\boldsymbol{\tau}]$. Given the delays satisfying $\max _{(m, n) \in \mathcal{L}} \tau_{(k, n)}<$ $\min _{m, d \in \mathcal{N}} \tau_{<k, d>}$, traffic $\mathbf{A}[t]$ is supportable if and only if

$$
\mathbf{E}[\mathbf{A}[t]] \in \Lambda_{\tau} .
$$

Proof: First if $\mathbf{A}[t]$ is supportable, then there exists a policy $\mathcal{P}$, under which the network is stochastically stable. From Lemma 4, we can find a corresponding $\mathcal{P}^{s}$ which allocates the same amount service rate to each link. Note that

$$
\lim _{t \rightarrow \infty} \mathbf{E}\left[\mathbf{S}^{\mathcal{P}_{s}}[t] \mid \mathbf{C}[t-\tau]=\mathbf{c}\right] \in \eta(\mathbf{C}[0]=\mathbf{c}) .
$$

According to the definition of $\Lambda_{\tau}$, it is easy to see that $\mathbf{E}[\mathbf{A}[t]] \in \Lambda_{\tau}$ if $\mathbf{A}[t]$ can be supported by the time-sharing policy $\mathcal{P}^{s}$.

The other direction is immediate. In particular, given the arrival rates, we can define a channel state dependent timesharing rule over appropriate vertices of $\Lambda_{\tau}$ that will stabilize (support) the process $\mathbf{A}[t]$ (see [3] Theorem 1 for an analogous proof).

\section{B. Throughput Optimality}

In this subsection, we prove that the on-off routing/scheduling is throughput optimal.

Theorem 1: Given the delays satisfying $\max _{(m, n) \in \mathcal{L}} \tau_{(m, n)}<$ $\min _{m, d \in \mathcal{N}} \tau_{<m, d>}$ and traffic $\mathbf{A}[t]$ such that $(1+\epsilon) \mathbf{A}[t]$ is supportable, the network is stochastically stable under the on-off routing/scheduling algorithm.

Proof: Define a Lyapunov function $V[t]$ such that

$$
V[t]=\sum_{m, d \in \mathcal{N}} Q_{<m, d>}^{2}[t]
$$

so we have

$$
\begin{aligned}
& \mathbf{E}[V[t+1]-V[t] \mid \mathbf{C}[t-\tau], \mathbf{Q}[t-\tau]] \\
&=\mathbf{E}\left[\sum_{m, d}\left(Q_{<m, d>}[t+1]-Q_{<m, d>}[t]\right) \times\right. \\
&\left.\left(Q_{<m, d>}[t+1]+Q_{<m, d>}[t]\right) \mid \mathbf{C}[t-\boldsymbol{\tau}], \mathbf{Q}[t-\boldsymbol{\tau}]\right] .
\end{aligned}
$$

Define $\Delta Q_{<m, d>}[t]=Q_{<m, d>}[t+1]-Q_{<m, d>}[t]$, we have that

$$
\begin{gathered}
\mathbf{E}[V[t+1]-V[t] \mid \mathbf{C}[t-\boldsymbol{\tau}], \mathbf{Q}[t-\boldsymbol{\tau}]] \\
=\mathbf{E}\left[\sum_{m, d} 2 \Delta Q_{<m, d>}[t] Q_{<m, d>}\left[t-\tau_{<m, d>}\right]+\right. \\
\Delta Q_{<m, d>}[t]\left(Q_{<m, d>}[t+1]+Q_{<m, d>}[t]-\right. \\
\left.\left.2 Q_{<m, d>}\left[t-\tau_{<m, d>}\right]\right) \mid \mathbf{C}[t-\boldsymbol{\tau}], \mathbf{Q}[t-\boldsymbol{\tau}]\right] .
\end{gathered}
$$

Note that $\Delta Q_{<m, d>}[t]$ and $Q_{<m, d>}[t]-Q_{<m, d>}[t-$ $\left.\tau_{<m, d>}\right]$ are both bounded since $\mathbf{A}[t]$ and $\mathbf{C}[t]$ are bounded. Thus, there exists a constant $K$ such that inequality (7) holds, where $\mathcal{M}^{*}[t]$ and $d_{(m, n)}^{*}[t]$ are as defined in the onoff scheduling algorithm.

Since $(1+\epsilon) \mathbf{A}[t]$ is supportable, we have $(1+\epsilon) \mathbf{E}[\mathbf{A}[t]] \epsilon$ $\Lambda_{\tau}$, which implies that there exists $\{\overline{\boldsymbol{\eta}}(\mathbf{c}) \in \eta(\mathbf{C}[0]=\mathbf{c})\}_{\mathbf{c}}$ 


$$
\begin{aligned}
\mathbf{E}\left[S_{(m, n)}^{\mathcal{P}}[\infty]\right]= & \mathbf{E}\left[\mathbf{E}\left[C_{(m, n)}(0: 0) D_{(m, n)}^{\mathcal{P}}(\mathbf{Q}(\boldsymbol{\tau}, \boldsymbol{\tau}), \mathbf{C}(\boldsymbol{\tau}, \boldsymbol{\tau})) \mid \mathbf{Q}(\boldsymbol{\tau}, \boldsymbol{\tau}), \mathbf{C}(\boldsymbol{\tau}, \boldsymbol{\tau})\right]\right] \\
= & \mathbf{E}\left[D_{(m, n)}^{\mathcal{P}}(\mathbf{Q}(\boldsymbol{\tau}, \boldsymbol{\tau}), \mathbf{C}(\boldsymbol{\tau}, \boldsymbol{\tau})) \mathbf{E}\left[C_{(m, n)}(0: 0) \mid \mathbf{Q}(\boldsymbol{\tau}, \boldsymbol{\tau}), \mathbf{C}(\boldsymbol{\tau}, \boldsymbol{\tau})\right]\right] \\
= & \mathbf{E}\left[D_{(m, n)}^{\mathcal{P}}(\mathbf{Q}(\boldsymbol{\tau}, \boldsymbol{\tau}), \mathbf{C}(\boldsymbol{\tau}, \boldsymbol{\tau})) \mathbf{E}\left[C_{(m, n)}(0: 0) \mid C_{(m, n)}\left(\tau_{(m, n)}: \tau_{(m, n)}\right)\right]\right] \\
= & \mathbf{E}\left[\mathbf{E}\left[C_{(m, n)}(0: 0) \mid C_{(m, n)}\left(\tau_{(m, n)}: \tau_{(m, n)}\right)\right] \mathbf{E}\left[D_{(m, n)}^{\mathcal{P}}(\mathbf{Q}(\boldsymbol{\tau}, \boldsymbol{\tau}), \mathbf{C}(\boldsymbol{\tau}, \boldsymbol{\tau})) \mid \mathbf{C}(\boldsymbol{\tau}: \boldsymbol{\tau})\right]\right] \\
={ }_{(b)} & \mathbf{E}\left[\mathbf{E}\left[C_{(m, n)}(0: 0) \mid C_{(m, n)}\left(\tau_{(m, n)}: \tau_{(m, n)}\right)\right] \mathbf{E}\left[D_{(m, n)}^{\mathcal{P}_{s}}(\mathbf{C}(\boldsymbol{\tau}, \boldsymbol{\tau})) \mid \mathbf{C}(\boldsymbol{\tau}: \boldsymbol{\tau})\right]\right] \\
= & \mathbf{E}\left[S_{(m, n)}^{\mathcal{P}_{s}}[\infty]\right]
\end{aligned}
$$

$$
\begin{aligned}
& \mathbf{E}[V[t+1]-V[t] \mid \mathbf{C}[t-\boldsymbol{\tau}], \mathbf{Q}[t-\boldsymbol{\tau}]] \\
\leq & K+2 \sum_{m, d} Q_{<m, d>}\left[t-\tau_{<m, d>}\right]\left(a_{[m, d]} 1_{[m, d] \in \mathcal{F}}+\sum_{(k, m) \in \mathcal{L}} \mathbf{E}\left[C_{(k, m)}[t] \mid C_{(k, m)}\left[t-\tau_{(k, m)}\right]\right] 1_{(k, m) \in \mathcal{M}^{*}[t], d_{(k, m)}^{*}[t]=d}\right. \\
& \left.-\sum_{(m, n) \in \mathcal{L}} \mathbf{E}\left[C_{(m, n)}[t] \mid C_{(m, n)}\left[t-\tau_{(m, n)}\right]\right] 1_{(m, n) \in \mathcal{M}^{*}[t], d_{(m, n)}^{*}[t]=d}\right) \\
= & K+2 \sum_{m, d} Q_{<m, d>}\left[t-\tau_{<m, d>}\right] a_{[m, d]} 1_{[m, d] \in \mathcal{F}}+ \\
& 2 \sum_{(m, n), d}\left(Q_{<m, d>}\left[t-\tau_{<m, d>}\right]-Q_{<n, d>}\left[t-\tau_{<n, d>}\right]\right) \mathbf{E}\left[C_{(m, n)}[t] \mid C_{(m, n)}\left[t-\tau_{(m, n)}\right]\right] 1_{(m, n) \in \mathcal{M}^{*}[t], d_{(m, n)}^{*}[t]=d}
\end{aligned}
$$

such that

$$
\begin{array}{r}
\sum_{\mathbf{c} \in \mathcal{C}|\mathcal{L}|} \operatorname{Pr}(\mathbf{C}[t-\boldsymbol{\tau}]=\mathbf{c})\left(\sum_{d \in \mathcal{N}}(1+\epsilon) a_{[m, d]} 1_{[m, d] \in \mathcal{F}}+\right. \\
\left.\sum_{(k, m) \in \mathcal{L}} \bar{\eta}_{(k, m)}(\mathbf{c})-\sum_{(m, n) \in \mathcal{L}} \bar{\eta}_{(m, n)}(\mathbf{c})\right) \leq 0
\end{array}
$$

for all $m, d \in \mathcal{N}$, which leads to inequality (9).

From the definition of the on-off scheduling algorithm, we further have inequality (10) holds for any given $\mathbf{C}[t-\tau]$.

Then, taking expectation on both sides of equality (9) over $\mathbf{C}[t-\boldsymbol{\tau}]$, and from inequalities (8) and (10), we can conclude that

$$
\begin{gathered}
\mathbf{E}[V[t+1]-V[t] \mid \mathbf{Q}[t-\boldsymbol{\tau}]] \\
\leq-2 \epsilon \sum_{m, d} Q_{<m, d>}\left[t-\tau_{<m, d>}\right] a_{[m, d] \in \mathcal{F}}+K,
\end{gathered}
$$

which implies that

$$
\begin{aligned}
& \mathbf{E}[V[t+1]-V[t]] \\
& \leq-2 \epsilon \mathbf{E}\left[\sum_{m, d} a_{[m, d]} Q_{<m, d>}\left[t-\tau_{<m, d>}\right] 1_{[m, d] \in \mathcal{F}}\right]+K .
\end{aligned}
$$

Thus, we can conclude that

$$
\begin{aligned}
& \lim \sup _{T \rightarrow \infty} \frac{1}{T} \sum_{t=0}^{T} \mathbf{E}\left[\sum_{[m, d] \in \mathcal{F}} Q_{<m, d>}\left[t-\tau_{<m, d>}\right] 1_{[m, d] \in \mathcal{F}}\right] \\
& \leq \frac{K+V[0]-\lim _{T \rightarrow \infty} V[T]}{2 \epsilon \max _{[m, d]} a_{[m, d]}}<\infty,
\end{aligned}
$$

and the theorem holds.

\section{Decentralized Routing/Scheduling: Detailed ANALYSIS}

In this section, we study the decentralized routing/scheduling. We only present the detailed analysis for NSI structure $S 2$ since the analysis of NSI structure $S 1$ is similar.

\section{A. Throughput Region}

First, given a threshold vector $\mathbf{T}=\left\{T_{(m, n)}\right\}_{(m, n) \in \mathcal{L}}$ and a probability vector $\mathbf{p}=\left\{p_{(m, n)}\right\}_{(m, n) \in \mathcal{L}}$, we define a threshold policy $\mathcal{T}(\mathbf{T}, \mathbf{p})$ as follows:

Threshold Policy $\mathcal{T}(\mathbf{T}, \mathbf{p})$ :

(i) If $C_{(m, n)}[t]>T_{(m, n)}$, node $m$ transmits over link $(m, n)$ at time slot $t$.

(ii) If $C_{(m, n)}[t]=T_{(m, n)}$, node $m$ transmits over link $(m, n)$ with probability $p_{(m, n)}$ at time slot $t$.

(iii) If $C_{(m, n)}[t]<T_{(m, n)}$, node $m$ keeps silent at time slot $t$.

Next, we denote by $S_{(m, n)}(\mathcal{T}(\mathbf{T}, \mathbf{p}))[t]$ the achievable rate over link $(m, n)$ at time slot $t$ under the threshold policy $\mathcal{T}(\mathbf{T}, \mathbf{p})$. Note that $S_{(m, n)}(\mathcal{T}(\mathbf{T}, \mathbf{p}))[t]=C_{(m, n)}[t]$ if no link in $\mathcal{I}_{(m, n)}$ is active simultaneously; and $S_{(m, n)}(\mathcal{T}(\mathbf{T}, \mathbf{p}))[t]=$ 0 otherwise. Given $\mathbf{C}[t-\tau]=\mathbf{c}$, the expected achievable rate over link $(m, n)$ is

$$
\mathbf{E}\left[S_{(m, n)}(\mathcal{T}(\mathbf{T}, \mathbf{p}))[t] \mid \mathbf{C}[t-\tau]=\mathbf{c}\right] .
$$

Then, we define $\boldsymbol{\eta}(\mathbf{c})$ to be the convex hull of $\mathbf{E}[\mathbf{S}(\mathcal{T}(\mathbf{T}, \mathbf{p}))[t] \mid \mathbf{C}[t-\tau]=\mathbf{c}]$ over all $\mathbf{T}$ and $\mathbf{p}$, i.e.,

$$
\boldsymbol{\eta}(\mathbf{c})=\mathcal{C H}_{\mathbf{T}, \mathbf{p}}(\mathbf{E}[\mathbf{S}(\mathcal{T}(\mathbf{T}, \mathbf{p}))[t] \mid \mathbf{C}[t-\tau]=\mathbf{c}]) .
$$




$$
\begin{aligned}
& \mathbf{E}[V[t+1]-V[t] \mid \mathbf{C}[t-\boldsymbol{\tau}], \mathbf{Q}[t-\boldsymbol{\tau}]] \\
\leq & K+2 \sum_{m, d} Q_{<m, d>}\left[t-\tau_{<m, d>}\right]\left(a_{[m, d]} 1_{[m, d] \in \mathcal{F}}+\sum_{(k, m) \in \mathcal{L}} \bar{\eta}_{(k, m)}(\mathbf{C}[t-\boldsymbol{\tau}])-\sum_{(m, n) \in \mathcal{L}} \bar{\eta}_{(m, n)}(\mathbf{C}[t-\boldsymbol{\tau}])\right)+ \\
& 2 \sum_{(m, n), d}\left(\mathbf{E}\left[C_{(m, n)}[t] \mid C_{(m, n)}\left[t-\tau_{(m, n)}\right]\right] 1_{(m, n) \in \mathcal{M}^{*}[t], d_{(m, n)}^{*}[t]=d}-\bar{\eta}_{(m, n)}(\mathbf{C}[t-\boldsymbol{\tau}])\right) \times \\
\left(Q_{<m, d>}\left[t-\tau_{<m, d>}\right]-Q_{<n, d>}\left[t-\tau_{<n, d>}\right]\right) &
\end{aligned}
$$

$$
\begin{gathered}
\sum_{(m, n), d}\left(\mathbf{E}\left[C_{(m, n)}[t] \mid C_{(m, n)}\left[t-\tau_{(m, n)}\right]\right] 1_{(m, n) \in \mathcal{M}^{*}[t], d_{(m, n)}^{*}[t]=d}-\bar{\eta}_{(m, n)}(\mathbf{C}[t-\boldsymbol{\tau}])\right) \times \\
\left(Q_{<m, d>}\left[t-\tau_{<m, d>}\right]-Q_{<n, d>}\left[t-\tau_{<n, d>}\right]\right) \leq 0 .
\end{gathered}
$$

Furthermore, we define $\tilde{\Lambda}_{\tau}$ such that

$$
\tilde{\Lambda}_{\tau}=\left\{\boldsymbol{\eta}: \boldsymbol{\eta}=\sum_{\mathbf{c} \in \mathcal{C}^{|\mathcal{L}|}} \operatorname{Pr}(\mathbf{C}[t-\tau]=\mathbf{c}) \boldsymbol{\eta}_{\mathbf{c}}, \boldsymbol{\eta}_{\mathbf{c}} \in \boldsymbol{\eta}(\mathbf{c})\right\} .
$$

Next define

$$
\mathbf{Y}[t]=\left(\mathbf{C}[t]\left(0: \tau+\tau_{f}\right), \mathbf{Q}[t]\left(0: \tau+\tau_{f}\right)\right)
$$

In the following lemma, we show that $\mathbf{Y}[t]$ is Markovian.

Lemma 6: Assume queues evolve as described in (5), then $\mathbf{Y}[t]$ is Markovian.

Proof: First, it is easy to see that

$$
\begin{aligned}
\operatorname{Pr}(\mathbf{C}[t+1] \mid \mathbf{Y}[t](0: t)) & =\operatorname{Pr}(\mathbf{C}[t+1] \mid \mathbf{C}[t]) \\
& =\operatorname{Pr}(\mathbf{C}[t+1] \mid \mathbf{Y}[t]) .
\end{aligned}
$$

Next, from queue dynamics (5), we can see that $\mathbf{Q}[t+1]$ is a function of $\mathbf{A}[t], \mathbf{Q}[t]$, and $\mathbf{S}\left[t-\tau_{f}\right]$. Note that $\mathbf{A}[t]$ is independent across time, $\mathbf{Q}[t]$ is included in $\mathbf{Y}[t]$, and $\mathbf{S}\left[t-\tau_{f}\right]$ is determined by $\mathbf{Q}[t]\left(\tau_{f}: \tau_{f}+\tau\right)$ and $\mathbf{C}[t]\left(\tau_{f}: \tau_{f}+\tau\right)$, which are also included in $\mathbf{Y}[t]$. Thus, we can conclude that

$$
\operatorname{Pr}(\mathbf{Q}[t+1] \mid \mathbf{Y}[t](0: t))=\operatorname{Pr}(\mathbf{Q}[t+1] \mid \mathbf{Y}[t]) \text {. }
$$

From equations (12) and (13), we can conclude that

$$
\operatorname{Pr}(\mathbf{Y}[t+1] \mid \mathbf{Y}[t](0: t))=\operatorname{Pr}(\mathbf{Y}[t+1] \mid \mathbf{Y}[t]),
$$

and the lemma holds.

In the next lemma, we will show that given traffic $\mathbf{A}[t]$, if the network can be stabilized by a scheduling algorithm $\mathcal{P}$, then there exists a time-sharing policy $\mathcal{P}_{s}$ that stabilizes the network as well. This is analogous to the development of the time-sharing policy in Section VI. To demonstrate this, we first define a time-sharing policy for a given stabilizing $\mathcal{P}$.

Note that if $\mathbf{A}[t]$ is supportable under $\mathcal{P}$, then $\mathbf{Y}^{\mathcal{P}}[t]$ is positive recurrent. Thus, we can define

$$
\begin{array}{cc} 
& \left(\mathbf{C}\left(0: \tau+\tau_{f}\right), \mathbf{Q}^{\mathcal{P}}\left(0: \tau+\tau_{f}\right)\right) \\
\triangleq & \lim _{t \rightarrow \infty}\left(\mathbf{C}[t]\left(0: \tau+\tau_{f}\right), \mathbf{Q}^{\mathcal{P}}[t]\left(0: \tau+\tau_{f}\right)\right) \\
= & \lim _{t \rightarrow \infty} \mathbf{Y}^{\mathcal{P}}[t] .
\end{array}
$$

Next we define a time-sharing policy related to $\mathcal{P}$.
Time-sharing Policy $\mathcal{P}_{s}$ : Given delayed information $\mathbf{C}[t-$ $\tau]=\mathbf{c}$, we let

$$
r_{\mathbf{q} \mid \mathbf{c}}^{\mathcal{P}}=\operatorname{Pr}\left(\mathbf{Q}^{\mathcal{P}}(0: \tau)=\mathbf{q}(0: \tau) \mid \mathbf{C}(\tau: \tau)=\mathbf{c}\right) .
$$

Then, at each time when the delayed channel state is $\mathbf{C}[t-\tau]=$ c, the policy $\mathcal{P}^{s}$ probabilistically make decisions (denoted by $\mathcal{D}^{\mathcal{P}_{s}}$ ) as follows: Let $\mathcal{Q}=\left\{\mathbf{q}: r_{\mathbf{q} \mid \mathbf{c}}^{\mathcal{P}}>0\right\}$. The policy $\mathcal{P}^{s}$ selects a $\mathbf{q} \in \mathcal{Q}$ with probability $r_{\mathbf{q} \mid \mathbf{c}}^{\mathcal{P}}$, and makes the corresponding decision be given by

$$
\begin{aligned}
& \mathbf{D}^{\mathcal{P}_{s}}(\mathbf{C}[t](0: \tau-1), \mathbf{C}[t-\tau]=\mathbf{c})= \\
& \mathbf{D}^{\mathcal{P}}(\mathbf{C}[t](0: \tau-1), \mathbf{C}[t-\tau]=\mathbf{c}, \mathbf{Q}[t](0: \tau)=\mathbf{q}(0: \tau))
\end{aligned}
$$

Note that this time-sharing policy is analogous to that described in Section VI, in the sense that it generates the decisions using a known stabilizing policy $\mathcal{P}$ by "feeding" it inputs which are randomly chosen (according to the stationary conditional distribution $r_{\mathbf{q} \mid \mathbf{c}}^{\mathcal{P}}$ ).

Example: Consider the two-user example studied in Section $\mathrm{V}-\mathrm{A}$, where, at each of the nodes, there is one-time-slot delay in the channel and queue state information from the other node. In this case, suppose that for given arrivals and channel model (i.e., Markov chain transition probabilities), a stabilizing queue and channel state based scheduling policy exists (denoted by $\mathcal{P}$ ) that makes transmission attempt decisions as described below. Note that $\mathcal{P}$ makes decision based on the common delayed NSI $\left(C_{(1,0)}[t-1], C_{(2,0)}[t-1]\right)$ and $\left(Q_{(1,0)}[t-1], Q_{(2,0)}[t-1]\right)$, along with the local current channel state information at each of the nodes ${ }^{4}$ )

For the delayed channel state $C_{(1,0)}[t-1]=C_{(2,0)}[t-1]=$ 50 , the stabilizing policy $\mathcal{P}$ makes decisions as follows:

(a) If $Q_{(1,0)}[t-1]>4 Q_{(2,0)}[t-1]$, node 1 attempts to transmit if its channel is non-zero (in this case, if $\left.C_{(1,0)}[t]=50\right)$, and remains silent if it's current channel is zero (i.e, $C_{(1,0)}[t]=0$ ). Node 2 remains silent irrespective of its current local channel state $\left(C_{(2,0)}[t]\right)$. Note that in this case, it is possible that neither of the nodes transmit even though a centralized scheduler

${ }^{4}$ While policy $\mathcal{P}$ can potentially use the current local queue length information at each of the nodes, in this example, it chooses not to do so. 
that knows all the channels at each time might make a transmission attempt (this happens when $\left(C_{(1,0)}[t]=\right.$ $\left.\left.0, C_{(2,0)}[t]=50\right)\right)$.

(b) If $Q_{(2,0)}[t-1]>4 Q_{(1,0)}[t-1]$, node 2 attempts to transmit and node 1 remains silent. The above decision happens irrespective of the current local channel states.

(c) Finally, if $Q_{(1,0)}[t-1] / Q_{(2,0)}[t-1] \in[0.25,4]$, node $i$ $(i=1,2)$ attempts to transmit if $C_{(i, 0)}[t]=50$. In this case, a collision could occur if both the (local) channels are at state 50 .

Similarly, suppose that for each of the other cases of delayed channel state (i.e., for each possible tuple of values of the random variables $\left.\left(C_{(1,0)}[t-1], C_{(2,0)}[t-1]\right)\right)$, the policy $\mathcal{P}$ can be described.

Further, suppose that under this policy $\mathcal{P}$ the following holds in steady-state (stationarity):

$$
\begin{aligned}
\operatorname{Pr}\left(\frac{Q_{(1,0)}^{\mathcal{P}}(1: 1)}{Q_{(2,0)}^{\mathcal{P}}(1: 1)}>4 \mid \mathbf{C}(1: 1)=(50,50)\right) & =0.3 \\
\operatorname{Pr}\left(\frac{Q_{(1,0)}^{\mathcal{P}}(1: 1)}{Q_{(2,0)}^{\mathcal{P}}(1: 1)}<\frac{1}{4} \mid \mathbf{C}(1: 1)=(50,50)\right) & =0.3 \\
\operatorname{Pr}\left(\frac{Q_{(2,0)}^{\mathcal{P}}(1: 1)}{Q_{(1,0)}^{\mathcal{P}}(1: 1)} \in[0.25,4] \mid \mathbf{C}(1: 1)=(50,50)\right) & =0.4 .
\end{aligned}
$$

Similarly, we can describe the conditional probabilities for other values of the delayed channel states. We recall that in the context of this example, $\mathbf{C}(1: 1)$ corresponds to the channel state of the pair of users with one-time-slot delay under system stationarity. Then, the corresponding time-sharing policy is as follows:

Time-sharing Policy: For each channel state $\left(C_{(1,0)}[t-\right.$ 1], $\left.C_{(2,0)}[t-1]\right)$, the time-sharing policy $\mathcal{P}_{s}$ will make a randomized decision depending on the decision taken by $\mathcal{P}$.

Suppose that $C_{(1,0)}[t-1]=C_{(2,0)}[t-1]=50$. Then, under the time-sharing policy $\mathcal{P}_{s}$,

(a) With probability 0.3 , node 1 attempts to transmit and node 2 keeps silent.

(b) With probability 0.3 , node 2 attempts to transmit and node 1 keeps silent.

(c) With probability 0.4 , both nodes $(i=1,2)$ attempt to transmit if $C_{(i, 0)}[t]=50$ (note that in this case, collision could occur).

Similarly, the time-sharing policy can be described for various other values of the delayed channel states.

In the following lemma, we will show that the link rates under $\mathcal{P}$ is the same as the service rate obtained under the corresponding $\mathcal{P}_{s}$.

Lemma 7: Given policy $\mathcal{P}$ which stabilizes the network with traffic $\mathbf{A}[t]$, we have that

$$
\lim _{t \rightarrow \infty} \mathbf{E}\left[S_{(m, n)}^{\mathcal{P}}[t]\right]=\lim _{t \rightarrow \infty} \mathbf{E}\left[S_{(m, n)}^{\mathcal{P}_{s}}[t]\right],
$$

where $\mathcal{P}_{s}$ is the corresponding time-sharing policy.

Proof: First, we can obtain equation (15), where the second equality yields from the definition of the time-sharing policy.
Note that $Q_{(m, n)}[t]$ is a function of $A_{(m, n)}[t](0: t)$ and $S_{(m, n)}[t]\left(0: \tau_{f}\right)$, and $S_{(m, n)}[t-\tilde{\tau}]$ is determined by $\mathbf{Q}[t-$ $\tilde{\tau}](0: \tau)$ and $\mathbf{C}[t-\tilde{\tau}](0: \tau)$. Since $\tau_{f}>\tau$, we can conclude that, conditioned on $C_{(m, n)}[t-\tau], C_{(m, n)}[t-r](r \leq \tau)$ is independent of $Q_{(l, k)}[s]$ for any $(l, k) \in \mathcal{L}$ and $s \leq t$. In other words, under policy $\mathcal{P}$,

$$
\begin{aligned}
& \operatorname{Pr}\left(\mathbf{C}(0: \tau-1) \mid \mathbf{C}(\tau: \tau), \mathbf{Q}^{\mathcal{P}}(0: \tau)\right) \\
= & \operatorname{Pr}(\mathbf{C}(0: \tau-1) \mid \mathbf{C}(\tau: \tau)) .
\end{aligned}
$$

Thus, we can further obtain that

$$
\lim _{t \rightarrow \infty} \mathbf{E}\left[S_{(m, n)}^{\mathcal{P}_{s}}[t] \mid \mathbf{C}[t-\tau]\right]=\lim _{t \rightarrow \infty} \mathbf{E}\left[S_{(m, n)}^{\mathcal{P}_{s}}[t] \mid \mathbf{C}[t-\tau]\right]
$$

as demonstrated in equality (17), where the last equality holds due to equation (16).

Now, give $\left(\mathbf{Q}^{\mathcal{P}}(0: \tau)=\mathbf{q}(0: \tau), \mathbf{C}(\tau: \tau)=\mathbf{c}\right)$, there exist $\mathbf{T}(\mathbf{c}, \mathbf{q}(0: \tau))$ and $\mathbf{p}(\mathbf{c}, \mathbf{q}(0: \tau))$ such that equality (18) holds for all $(m, n) \in \mathcal{L}$. In other words, there exist $\mathbf{T}(\mathbf{c}, \mathbf{q}(0: \tau))$ and $\mathbf{p}(\mathbf{c}, \mathbf{q}(0: \tau))$ such that, given the queue lengths and delayed channel states, the probability the channel state of $(m, n)$ is greater than or equal to the threshold is the same as the probability that link $(m, n)$ is active under the time-sharing policy. Then, we can further define a thresholdbased time-sharing policy.

Threshold-Based Time-sharing Policy $\mathcal{P}_{t s}$ : Given delayed channel-states $\mathbf{C}[t-\tau]=\mathbf{c}$, we let

$$
r_{\mathbf{q} \mid \mathbf{c}}^{\mathcal{P}}=\operatorname{Pr}\left(\mathbf{Q}^{\mathcal{P}}(0: \tau)=\mathbf{q}(0: \tau) \mid \mathbf{C}(\tau: \tau)=\mathbf{c}\right) .
$$

Then, at each time when the delayed channel state is $\mathbf{C}[t-\tau]=$ c, the policy $\mathcal{P}_{t s}$ probabilistically make decisions (denoted by $\mathcal{D}^{\mathcal{P}_{t s}}$ ) as follows: Let $\mathcal{Q}=\left\{\mathbf{q}: r_{\mathbf{q} \mid \mathbf{c}}^{\mathcal{P}}>0\right\}$. Policy $\mathcal{P}_{t s}$ chooses $\mathbf{q} \in \mathcal{Q}$, with probability $r_{\mathbf{q} \mid \mathbf{c}}^{\mathcal{P}}$, and lets the corresponding decision be given by

$$
\begin{gathered}
D_{(m, n)}^{\mathcal{P}_{t s}}\left(C_{(m, n)}[t](0: \tau-1), \mathbf{C}[t-\tau]=\mathbf{c}\right) \\
=1_{C_{(m, n)}[t]>T_{(m, n)}(\mathbf{c}, \mathbf{q}(0: \tau))}+z 1_{C_{(m, n)}[t]=T_{(m, n)}(\mathbf{c}, \mathbf{q}(0: \tau))},
\end{gathered}
$$

where

$$
\operatorname{Pr}(z=1)=p_{(m, n)}(\mathbf{c}, \mathbf{q}(0: \tau))
$$

In other words, for each delayed channel state $\mathbf{c}$, the policy $\mathcal{P}_{t s}$ first randomly (with probability $r_{\mathbf{q} \mid \mathbf{c}}^{\mathcal{P}}$ ) picks a possible stationary queue length under policy $\mathcal{P}$. Then the policy uses the resulting pair $(\mathbf{q}, \mathbf{c})$ to determine the threshold $T_{(m, n)}(\mathbf{c}, \mathbf{q})$ and the probability $p_{(m, n)}(\mathbf{c}, \mathbf{q})$, and uses the threshold and the probability to make the transmission decision.

In the following lemma, we will show that considering $\mathcal{P}_{s}$ and $\mathcal{P}_{t s}$, which are related to the same stabilizing policy $\mathcal{P}$, the service rate obtained by link $(m, n)$ under the threshold time-sharing policy is no less than the one under the time sharing policy.

Lemma 8: Assuming that $\mathcal{P}_{s}$ and $\mathcal{P}_{t s}$ are the time-sharing policy and the threshold-based time-sharing policy defined based the stabilizing policy $\mathcal{P}$, then

$$
\lim _{t \rightarrow \infty} \mathbf{E}\left[S_{(m, n)}^{\mathcal{P}_{s}}[t]\right] \leq \lim _{t \rightarrow \infty} \mathbf{E}\left[S_{(m, n)}^{\mathcal{P}_{t s}}[t]\right] .
$$




$$
\begin{aligned}
& \mathbf{E}\left[S_{(m, n)}^{\mathcal{P}_{s}}[t] \mid \mathbf{C}[t-\tau]=\mathbf{c}\right] \\
&= \mathbf{E}\left[C_{(m, n)}[t] D_{(m, n)}^{\mathcal{P}_{s}}\left(C_{(m, n)}[t](0: \tau-1), \mathbf{C}[t-\tau]\right) \times\right. \\
&\left.\prod_{(l, k) \in \mathcal{I}_{(m, n)}}\left(1-D_{(l, k)}^{\mathcal{P}_{s}}\left(C_{(l, k)}[t](0: \tau-1), \mathbf{C}[t-\tau]\right)\right) \mid \mathbf{C}[t-\tau]=\mathbf{c}\right] \\
&=\sum_{\mathbf{q}(0: \tau)} \operatorname{Pr}\left(\mathbf{Q}^{\mathcal{P}}(0: \tau)=\mathbf{q}(0: \tau) \mid \mathbf{C}(\tau: \tau)=\mathbf{c}\right) \times \\
& \mathbf{E}\left[C_{(m, n)}[t] D_{(m, n)}^{\mathcal{P}}\left(C_{(m, n)}[t](0: \tau-1), \mathbf{C}[t-\tau], \mathbf{Q}[t](0: \tau)=\mathbf{q}(0: \tau)\right) \times\right. \\
&\left.\prod_{(l, k) \in \mathcal{I}_{(m, n)}}\left(1-D_{(l, k)}^{\mathcal{P}}\left(C_{(l, k)}[t](0: \tau-1), \mathbf{C}[t-\tau], \mathbf{Q}[t](0: \tau)=\mathbf{q}(0: \tau)\right)\right) \mid \mathbf{C}[t-\tau]=\mathbf{c}\right]
\end{aligned}
$$

$$
\begin{aligned}
& \lim _{t \rightarrow \infty} \mathbf{E}\left[S_{(m, n)}^{\mathcal{P}_{s}}[t] \mid \mathbf{C}[t-\tau]=\mathbf{c}\right] \\
= & \sum_{\mathbf{q}(0: \tau)} \operatorname{Pr}\left(\mathbf{Q}^{\mathcal{P}}(0: \tau)=\mathbf{q}(0: \tau) \mid \mathbf{C}(\tau: \tau)=\mathbf{c}\right) \mathbf{E}\left[C_{(m, n)}(0: 0) D_{(m, n)}^{\mathcal{P}}\left(C_{(m, n)}(0: \tau-1), \mathbf{C}(\tau: \tau), \mathbf{Q}(0: \tau)\right) \times\right. \\
& \left.\quad \prod_{(l, k) \in \mathcal{I}_{(m, n)}}\left(1-D_{(l, k)}^{\mathcal{P}}\left(C_{(l, k)}(0: \tau-1), \mathbf{C}(\tau: \tau), \mathbf{Q}(0: \tau)\right)\right) \mid \mathbf{C}(\tau: \tau)=\mathbf{c}, \mathbf{Q}(0: \tau)=\mathbf{q}(0: \tau)\right] \\
= & \lim _{t \rightarrow \infty} \mathbf{E}\left[\mathbf{E}\left[S_{(m, n)}^{\mathcal{P}}[t] \mid \mathbf{Q}^{\mathcal{P}}(0: \tau)\right] \mid \mathbf{C}[t-\tau]=\mathbf{c}\right] \\
= & \lim _{t \rightarrow \infty} \mathbf{E}\left[S_{(m, n)}^{\mathcal{P}}[t] \mid \mathbf{C}[t-\tau]=\mathbf{c}\right]
\end{aligned}
$$

$$
\begin{aligned}
& \operatorname{Pr}\left(C_{(m, n)}(0: 0)>T_{(m, n)}(\mathbf{c}, \mathbf{q}(0: \tau)) \mid \mathbf{C}(\tau: \tau)=\mathbf{c}\right) \\
& +p_{(m, n)}(\mathbf{c}, \mathbf{q}(0: \tau)) \operatorname{Pr}\left(C_{(m, n)}(0: 0)=T_{(m, n)}(\mathbf{c}, \mathbf{q}(0: \tau)) \mid \mathbf{C}(\tau: \tau)=\mathbf{c}\right)= \\
& \operatorname{Pr}\left(D_{(m, n)}^{\mathcal{P}}\left(C_{(m, n)}(0: \tau-1), \mathbf{C}(\tau: \tau), Q_{<m, n>}(0: \tau-1)=q_{<m, n>}(0: \tau-1), \mathbf{Q}(\tau: \tau)=\mathbf{q}(\tau: \tau)\right)=1 \mid \mathbf{C}(\tau: \tau)=\mathbf{c}\right)
\end{aligned}
$$

$\mathbf{E}\left[C_{(m, n)}[t] 1_{C_{(m, n)}[t]>T_{(m, n)}(\mathbf{c}, \mathbf{q}(0: \tau))}+C_{(m, n)}[t] z 1_{C_{(m, n)}[t]>T_{(m, n)}(\mathbf{c}, \mathbf{q}(0: \tau))} \mid \mathbf{C}(\tau: \tau)=\mathbf{c}\right] \geq$

$\mathbf{E}\left[C_{(m, n)}[t] D_{(m, n)}^{\mathcal{P}}\left(C_{(m, n)}(0: \tau-1), \mathbf{C}(\tau: \tau), Q_{<m, n>}(0: \tau-1)=q_{<m, n>}(0: \tau-1), \mathbf{Q}(\tau: \tau)=\mathbf{q}(\tau: \tau)\right) \mid \mathbf{C}(\tau: \tau)=\mathbf{c}\right]$

Proof: From equality (18), we can obtain equation (19) holds for any link $(m, n)$. Combining (18) and (19), we can conclude that the lemma holds.

Lemma 9: Considering NSI structure $S 2$, traffic $\mathbf{A}[t]$ is supportable if and only if $\mathbf{A}[t] \in \tilde{\Lambda}_{\tau}$.

Proof: From Lemma 7 and 8, we can conclude that

$$
\mathbf{E}\left[A_{(m, n)}[\infty]\right] \leq \lim _{t \rightarrow \infty} \mathbf{E}\left[S_{(m, n)}^{\mathcal{P}_{t s}}[\infty]\right]
$$

if $\mathbf{A}[t]$ is supportable. From the definition of the thresholdbased time-sharing policy, we also have that

$$
\mathbf{E}\left[\mathbf{S}^{\mathcal{P}_{t s}}[\infty]\right] \in \tilde{\Lambda}_{\tau},
$$

which implies that $\mathbf{E}[\mathbf{A}[t]] \in \tilde{\Lambda}_{\tau}$ if $\mathbf{A}[t]$ is supportable.
Now assume $\mathbf{E}[\mathbf{A}[t]] \in \tilde{\Lambda}_{\tau}$, then from the definition of $\tilde{\Lambda}_{\tau}$, there exists a threshold time-sharing policy that stabilizes the network.

\section{B. Throughput Optimality}

Next we show the threshold routing/scheduling algorithm is throughput-optimal for case (ii).

Theorem 2 Given NSI structure $S 2$, and traffic $\mathbf{A}[t]$ such that $(1+\epsilon) \mathbf{E}[\mathbf{A}[t]] \in \tilde{\Lambda}_{\tau}$, the network is stochastically stable under the threshold-based routing/scheduling algorithm.

Proof: Define $\tilde{Q}_{<m, d>}[t]$ to be the number of packets that are queued at node $m$, but have not been delivered to node $d$. 
Define a Lyapunov function

$$
V[t]=\sum_{(m, n)} \tilde{Q}_{<m, n>}^{2}[t] .
$$

Similar to the analysis in Theorem 1, we can obtain that there exists $K>0$ such that

$$
\begin{aligned}
& \mathbf{E}[V[t+1]-V[t]] \\
\leq & -2 \epsilon \sum_{(m, n)} a_{(m, n)} \mathbf{E}\left[\tilde{Q}_{<m, n>}[t-\tau]\right] 1_{(m, n) \in \mathcal{F}}+K,
\end{aligned}
$$

and the theorem holds.

\section{CONCLUSION}

In this paper, we studied the impact of delayed NSI on network throughput. We provided the relations between the delays in NSI and the network throughput region. We also developed throughput-optimal scheduling algorithms that incorporate the delayed NSI, both in the context of centralized and decentralized scheduling. In the decentralized scenario, we characterized the impact for two special cases (homogeneous delayed NSI from other nodes, with flow/delay restrictions). In our future research, we will consider the decentralized routing/scheduling with heterogeneously delayed NSI and multihop traffic flows.

\section{ACKNOWLEDGMENT}

This work was partially supported by NSF Grants CNS0347400, CNS-0519535, and CNS-0721380, the Darpa ITMANET program and the DTRA grant HDTRA1-08-1-0016.

\section{REFERENCES}

[1] L. Tassiulas and A. Ephremides, "Stability properties of constrained queueing systems and scheduling policies for maximum throughput in multihop radio networks," IEEE Trans. Automat. Contr., vol. 4, pp. 1936-1948, December 1992.

[2] —-, "Dynamic server allocation to parallel queues with randomly varying connectivity," IEEE Trans. Inform. Theory, vol. 39, pp. 466478, March 1993.

[3] M. Andrews, K. Kumaran, K. Ramanan, A. Stolyar, R. Vijayakumar, and P. Whiting, "CDMA data QoS scheduling on the forward link with variable channel conditions," Bell Labs Tech. Memo, April 2000.

[4] A. Stolyar and K. Ramanan, "Largest weighted delay first scheduling: Large deviations and optimality," Adv. Appl. Prob., vol. 11, pp. 1-48, 2001.

[5] S. Shakkottai and A. Stolyar, "Scheduling for multiple flows sharing a time-varying channel: The exponential rule," Ann. Math. Statist., vol. 207, pp. 185-202, 2002.

[6] X. Lin and N. Shroff, "Joint rate control and scheduling in multihop wireless networks," Paradise Island, Bahamas, December 2004.

[7] S. Shakkottai, R. Srikant, and A. Stolyar, "Pathwise optimality of the exponential scheduling rule for wireless channels," Adv. Appl. Prob., vol. 36, no. 4, pp. 1021-1045, December 2004.

[8] A. Eryilmaz, R. Srikant, and J. Perkins, "Stable scheduling policies for fading wireless channels," IEEE/ACM Trans. Network., vol. 13, pp. 411424, 2005.

[9] A. Eryilmaz and R. Srikant, "Fair resource allocation in wireless networks using queue-length-based scheduling and congestion control," in Proc. IEEE Infocom., 2005.

[10] M. Neely, E. Modiano, and C. Li, "Fairness and optimal stochastic control for heterogeneous networks," in Proc. IEEE Infocom., vol. 3 , Miami, FL, March 2005, pp. 1723-1734.

[11] A. Stolyar, "Maximizing queueing network utility subject to stability: Greedy primal-dual algorithm," Queueing Systems, vol. 50, no. 4, pp. 401-457, August 2005.
[12] X. Lin and S. Rasool, "Constant-time distributed scheduling policies for ad hoc wireless networks," in Proc. Conf. on Decision and Control, 2006.

[13] X. Wu and R. Srikant, "Scheduling efficiency of distributed greedy scheduling algorithms in wireless networks," in Proc. IEEE Infocom., 2006.

[14] A. Eryilmaz, A. Ozdaglar, and E. Modiano, "Polynomial complexity algorithms for full utilization of multi-hop wireless networks," in Proc. IEEE Infocom., 2007.

[15] A. Gupta, X. Lin, and R. Srikant, "Low-complexity distributed scheduling algorithms for wireless networks," in Proc. IEEE Infocom., 2007.

[16] S. Sanghavi, L. Bui, and R. Srikant, "Distributed link scheduling with constant overhead," in Proc. Ann. ACM SIGMETRICS Conf., San Diego, CA, June 2007.

[17] C. Joo, X. Lin, and N. B. Shroff, "Understanding the capacity region of the greedy maximal scheduling algorithm in multi-hop wireless networks," in Proc. IEEE Infocom., Phoenix, Arizona, April 2008.

[18] L. Georgiadis, M. J. Neely, L. Tassiulas, "Resource Allocation and Cross-Layer Control in Wireless Networks," Foundations and Trends in Networking, Vol. 1, no. 1, pp. 1-144, 2006.

[19] X. Lin, N. Shroff, and R. Srikant, "A tutorial on cross-layer optimization in wireless networks," IEEE J. Sel. Areas Commun., 2006.

[20] X. Qin and R. Berry, "Opportunistic splitting algorithms for wireless networks," in Proc. IEEE Infocom., March 2004.

[21] — " "Opportunistic splitting algorithms for wireless networks with heterogenous users," in Proc. Conf. on Information Sciences and Systems (CISS), March 2004.

[22] S. Adireddy and L. Tong, "Exploiting decentralized channel state information for random access," IEEE Trans. Inform. Theory, no. 2, Feb. 2005.

[23] A. Gopalan, C. Caramanis, and S. Shakkottai, "On wireless scheduling with partial channel-state information," in Proc. Ann. Allerton Conf. Communication, Control and Computing, Urbana, IL, September 2007.

[24] D. Zheng, W. Ge, and J. Zhang, "Distributed opportunistic scheduling for ad-hoc communications: An optimal stopping approach," in $A C M$ Mobihoc, 2007.

[25] N. Chang and M. Liu, "Optimal channel probing and transmission scheduling for opportunistic spectrum access," in ACM Int. Conf. on Mobile Computing and Networking (MobiCom), September 2007.

[26] Z. Ji, Y. Yang, J. Zhou, M. Takai, and R. Bagrodia, "Exploiting medium access diversity in rate adaptive wireless LANs," in Proc. ACM MOBICOM, 2004.

[27] A. Sabharwal, A. Khoshnevis, and E. Knightly, "Opportunistic spectral usage: Bounds and a multi-band CSMA/CA protocol," IEEE/ACM Transactions on Networking, 2006.

[28] S. Guha, K. Munagala, and S. Sarkar, "Performance guarantees through partial information based control in multichannel wireless networks," University of Pennsylvania, Tech. Rep., 2006, http://www.seas.upenn.edu/ swati/report.pdf.

[29] A. Pantelidou, A. Ephremides, and A. L. Tits, "Joint scheduling and routing for ad-hoc networks under channel state uncertainty," in 5th Intl. Symposium on Modeling and Optimization in Mobile, Ad-Hoc and Wireless Networks (WiOpt), April 2007.

[30] K. Kar, X. Luo, and S. Sarkar, "Throughput-optimal scheduling in multichannel access point networks under infrequent channel measurements," in Proceedings of IEEE Infocom, Anchorage, AK, May 2007.

[31] L. Ying and S. Shakkottai, "On Throughput Optimality with Delayed Network-State Information," Proceedings of the 2008 Information Theory and Applications Workshop, San Diego, CA, February 2008.

[32] H. S. Witsenhausen, "A counter-example in stochastic optimal control," SIAM Journal on Control, Volume 6, 1968.

[33] H. S. Witsenhausen, "Information structures, feedback and causality," SIAM Journal on Control, Volume 9, 1972.

[34] N. Sandell and M. Athans, "Solution of some nonclassical lqg stochastic decision problems," IEEE Trans. Automat. Contr., no. 2, pp. 108-116, April 1974.

[35] P. Varaiya and J. Walrand, "On delayed sharing patterns," IEEE Trans. Automat. Contr., vol. 23, no. 3, pp. 443-445, June 1978. 